\title{
Marine bioactive fibers: alginate and chitosan fibers-a critical review
}

\begin{abstract}
Alginate and chitosan are two marine natural polymers with unique structures and properties. Alginate is a polymeric acid, whilst chitosan is a polymeric amine. Both polymers can be made into fibers through the wet-spinning process. This article reviews the novel properties of alginate and chitosan, and compares the functional performances of the two fibers as novel textile materials. The principal applications of alginate and chitosan fibers are also reviewed.
\end{abstract}

Keywords: alginate, chitosan, chemical fiber, biomaterial, bioactivity, medical textile, cosmetotextile

\author{
Volume I Issue 6 - 2017 \\ Yimin Qin, ${ }^{1,2}$ Yunlong Deng, ${ }^{2}$ Yuna $\mathrm{Hao},{ }^{2} \mathrm{Ni}$ \\ Zhang, ${ }^{2}$ Xianming Shang ${ }^{2}$ \\ 'College of Material and Textile Engineering, China \\ ${ }^{2}$ State Key Laboratory of Bioactive Seaweed Substances, China
}

Correspondence: Yimin Qin, College of Material and Textile Engineering, Jiaxing College, Jiaxing 3|400 I, Zhejiang Province, China, Email yiminqin 1965@126.com

Received: April 06, 2017| Published: May 15, 2017

\section{Introduction}

Alginate and chitosan are two natural polymers extracted from brown seaweeds and crustacean shells respectively. In recent years, both alginate and chitosan have found widespread uses as novel biomaterials. ${ }^{1}$ In particular, they have been made into fibers through the wet-spinning process and in line with their marine origin, these fibers are biocompatible and biodegradable, and possess excellent bioactivities that are highly valuable in the development of functional textile products such as medical textiles, hygiene products, cosmetotextiles and those textile materials that are in close contact with the body, such as under wears, bedding materials, etc. ${ }^{2-4}$ As renewable resources, both alginate and chitosan have abundant supplies in nature. ${ }^{5,6}$ The seaweeds used for alginate extraction can come from wild varieties as well as cultivated species, whilst chitosan can be extracted from seafood processing waste of crabs, shrimps, prawns, krills, etc. Figure 1 illustrates the raw materials for alginate and chitosan.

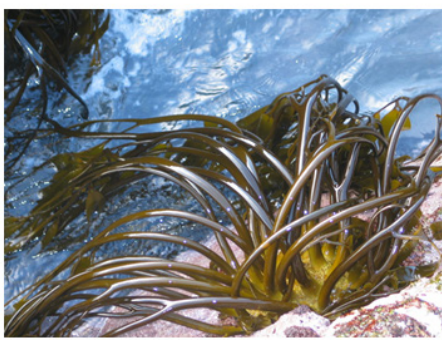

(I)

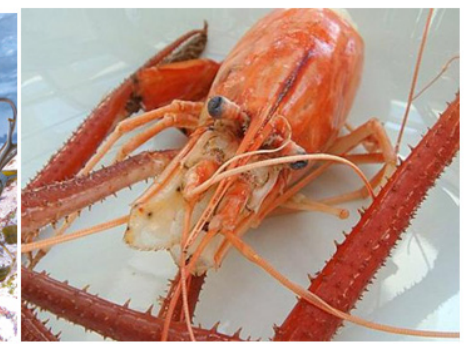

(2)
Figure I Raw materials for alginate and chitosan, (I) brown seaweed; (2) crustacean shell waste

\section{Fiber production}

Chemically, alginate is a polymeric acid composed of two types of monomer units, i.e., $\alpha$-L-guluronic acid $(\mathrm{G})$ and $\beta$-D-mannuronic acid (M). Depending on the type of seaweeds, alginate varies in molecular weight and the G, M, GG, MM and GM compositions. The G and $\mathrm{M}$ contents, and additionally the GG, MM and MG contents in the alginate have a significant effect on the properties of the alginate fibers. Chitosan is a polymeric amine. Its chemical structure is poly- $(1 \rightarrow 4)$ 2 -amino-2-deoxy- $\beta$-D-glucose. Commercially, chitosan is obtained by de acetylation of chitin, which is poly-(1 $\rightarrow 4)$-2-acetamino-2-deoxy$\beta$-D-glucose. Similar to the G/M contents in alginate, the degree of acetylation on the amine group is an important structural parameter for chitosan. Polymers with above $50 \%$ acetylation are considered as chitin while those with less than $50 \%$ acetylation are considered as chitosan. The chemical structures of alginic acid, chitin and chitosan are shown in Figure 2.
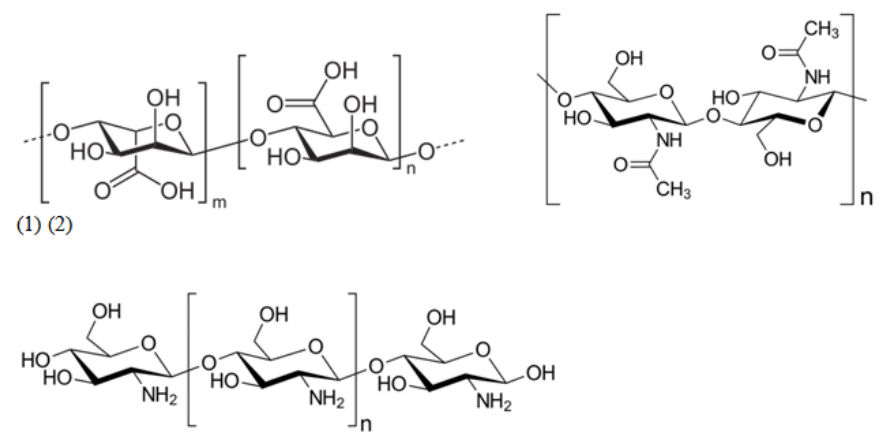

(3)

Figure 2 Chemical structures of (I) alginic acid; (2) chitin and (3) chitosan.

During the fiber making process, sodium alginate is dissolved in water and chitosan is dissolved in aqueous acetic acid solution to prepare their respective spinning solutions, which are then extruded via spinneret into coagulation baths containing $\mathrm{CaCl}_{2}$ and $\mathrm{NaOH}$ respectively to precipitate the two polymers into fibers. ${ }^{7,2}$ Upon washing, stretching and drying, alginate and chitosan fibers can be made with tensile properties similar to conventional viscose fibers, which can be processed in the many textile processes such as yarn spinning, weaving, knitting, and nonwoven. ${ }^{8,1}$ Figure 3 shows a schematic illustration of the wet spinning process.

As a natural polymeric acid, alginate is negatively charged when sodium alginate is dissolved in water, whilst chitosan is a positively charged polymer due to the amine groups in its structure. The ionic interaction between alginate and chitosan was utilized by Tamura et al. ${ }^{9}$ when preparing chitosan coated calcium alginate fibers, by extruding sodium alginate solution into a coagulation bath containing chitosan. Knill et al. ${ }^{10}$ improved on the method by first degrading chitosan into low molecular weight oligosaccharide, which can penetrate better into 
the swollen calcium alginate fibers. The resultant fibers were found to have high absorbency as well as sustained antimicrobial properties Miraftab et al. ${ }^{11}$ and Majima et al. ${ }^{12}$ found that the poly blend fibers made from alginate and chitosan have better biocompatibility and cell affinity, which are suitable for the preparation of scaffolds in tissue engineering.

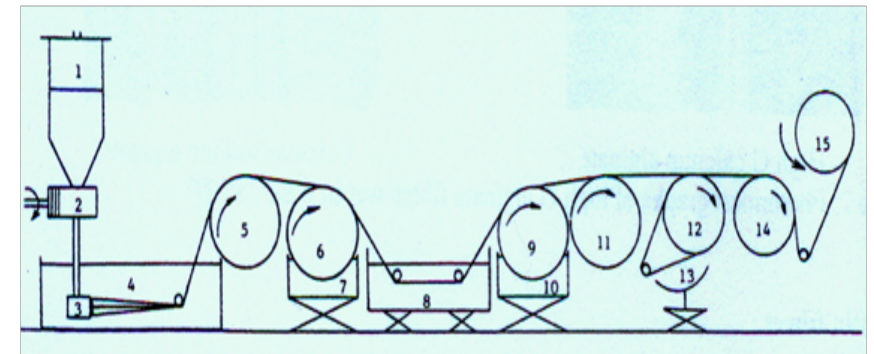

Figure 3 Schematic illustration of a small wet spinning production line, (I) spinning solution; (2) metering pump; (3) spinneret; (4) coagulation bath; (5) take-up roller I; (6) take-up roller II; (7) water bath; (8) hot water stretching bath; (9) stretching roller I; (I0) acetone washing bath; (II) stretching roller II; (I2) advancing roller I; (I3) heater; (I4) advancing roller II; (I5) winding up unit.

Alginate and chitosan based nano fibers can be made through the electro spinning process, in which alginate and chitosan solutions are deposited as fibrous material by charging the liquid to about $5-30 \mathrm{kV}$ and ejecting it through a nozzle onto an oppositely charged grounded target. The electro spinning system consists of a high-voltage DC supply, a grounded electrode, a nozzle system with diameter controls, and a fixed or rotated target to which the spun fiber could be adhered. Alginate and chitosan nano fibers can be made in this way with excellent surface morphology and porosity to provide the most appropriate interface for biomedical applications. The fibers are often spun onto nonwoven structures which are porous and have high surface area, with the resulting electrospun nonwoven structure ideally suited for making scaffolding for tissue engineering. ${ }^{1}$

\section{Fiber properties}

Alginate and chitosan fibers have many unique performance characteristics, some of them are summarized below.

High absorption capacity: As a polymeric acid, alginate has novel ion exchange property and high absorption capacity. In particular, when calcium alginate fibers are placed in contact with solutions containing sodium ions, such as the body fluid, calcium ions are released and sodium ions get into the fibers to form sodium alginate, which is soluble in water. As a result, a large amount of water is drawn into the fibers to form highly swollen fibrous gel, a property highly valuable in medical, hygiene and cosmetic textile materials. ${ }^{13,14}$ Figure 4 shows photomicrographs of alginate fibers before and after wetting in saline. Due to the hydroxyl and amine groups, chitosan fibers also have high affinity to water, with moisture regain at about $16 \%$. When combined with their antimicrobial properties, chitosan fibers are ideally suited to be blended into textile materials for underwear and bedding materials. Chitosan based face mask products are also gaining popularity in the cosmetic industry.

Strong binding with metal ions: As a polymeric acid, alginate can form salt with metal ions. Most divalent metal ions can form water insoluble salt with alginate, hence alginate fibers can be made by extruding water soluble sodium alginate solution into aqueous solutions containing divalent metal ions. ${ }^{15,16}$ Many metal ions are useful for wound healing. For example, zinc is useful for zinc deficient patients, whilst silver has anti-microbial properties. Sodium alginate can be extruded into an aqueous zinc chloride solution to form zinc alginate fibers. Alternatively, calcium alginate fibers can be treated with solutions containing zinc or silver and upon ion-exchange; zinc and silver ions in the solution replace calcium ions in the fibers, resulting in alginate fibers containing zinc and silver ions. Chitosan fibers can bind metal ions via chelation with the amine groups. It has been shown that chitosan fibers can chelate up to $6.2 \%$ by weight of zinc ions, with the resultant fiber highly antimicrobial against a broad spectrum of micro-organisms.

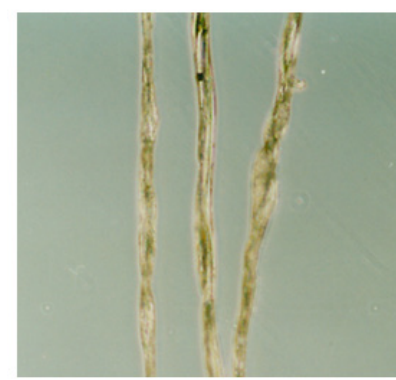

( 1$)$
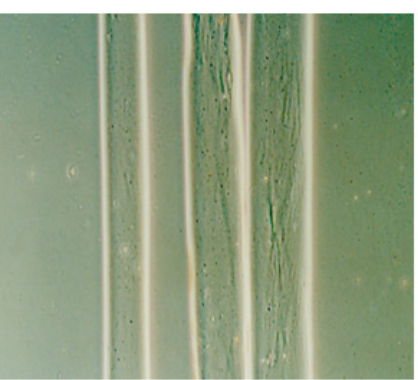

(2)
Figure 4 Photomicrographs of alginate fiber, (I) dry, (2) wet in saline.

Anti-microbial properties: Both alginate and chitosan fibers are known to have anti-microbial properties. When fluid is absorbed into an alginate based fibrous material, the fibers swell after the ion exchange process. As the fibers swell, the space between the fibers is closed and any bacteria that are carried in the fluid are trapped, thereby inhibiting the spreading of bacteria. The anti-microbial mechanism for chitosan is different from that of the alginate fibers. As a polymeric amine, chitosan is positively charged when wet. As the cell walls of the bacteria are negatively charged, bacteria are adhered to the chitosan fibers and due to their different electric charge, antimicrobial effect is achieved through the burst of cell walls.

\section{Applications of alginate and chitosan fibers}

Alginate and chitosan fibers have been used in a variety of applications; in particular, they are now widely used in cosmetic, medical and hygiene textile materials.

\section{Cosmeto textiles}

Cosmetotextiles are textile material with cosmetic properties, although these types of textiles can also harbor other functions and ingredients, such as medical properties, mosquito repellents, odor reducers, antimicrobials or UV-protection agents.

Cosmetic textile is an industry that has grown along with consumer interest in wellness and well-being. It involves the use of fiber and textile materials to deliver a wide range of microencapsulated ingredients such as aloe vera, vitamin E, retinol, and caffeine that can offer moisturizing, firming, or slimming benefits. ${ }^{17}$ The next generation of cosmetic textile products could potentially go beyond beauty, by utilizing innovative new methods to deliver medical, antiaging, and stress-relieving benefits through apparel textiles and other products. In this respect, alginate and chitosan fibers and nonwoven fabrics are highly hydrophilic and biocompatible, which are ideal materials for the production of face masks. In addition, the fibers and 
fabrics can be used to carry various bioactive ingredients to achieve sustained release to the skin. Figure 5 shows face mask made from hydro entangled alginate nonwoven fabric.

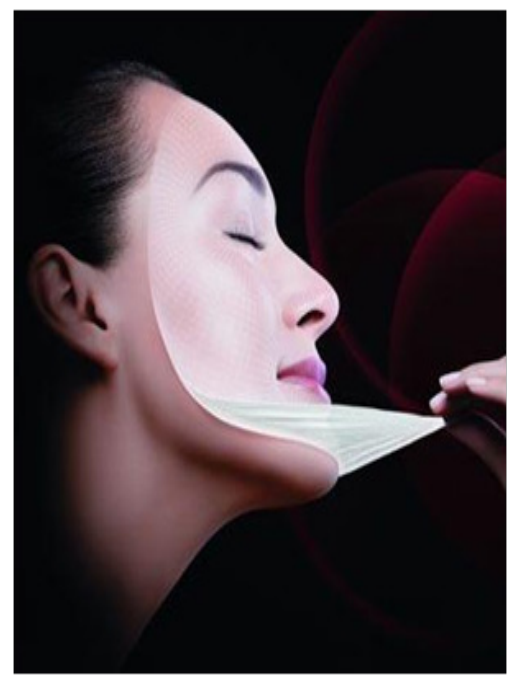

Figure 5 Face mask made from hydro entangled alginate nonwoven fabric.

\section{Functional wound dressings}

One of the main applications of alginate and chitosan fibers are in wound dressing materials. Since they are hydrophilic polymers, alginate and chitosan fibers are known to have high absorption capacities and are able to retain moisture. When on contact with wound exudates, alginate fibers and dressings have ion-exchange properties whereby calcium ions in the fibers are replaced by the sodium ions in the body fluid, resulting in the formation of a moist gel on the wound surface. Many studies have confirmed the unique wound healing properties of alginate wound dressings. ${ }^{18-20}$

In recent years, alginate fibers have been successfully used as a 'moist healing' material. Wound dressings made from alginate fibers have occupied a significant part of the high tech wound dressing market in Western Europe and North America. Similarly, chitosan fibers have novel wound healing properties and in recent years, much efforts have been made to use chitosan as a material for tissue engineering applications, utilizing its excellent biocompatibility and biodegradability.

\section{Hygiene products}

Over the past 50years, absorbent hygiene products such as baby diapers, adult incontinence products, feminine protection pads and personal care wipes have all become essential features of modern day life. Their increased use has been accompanied by dramatic improvements in skin health and hygiene; particularly in the incidence of diaper dermatitis. Alginate and chitosan fibers can be made into hydro entangled nonwoven fabric and used as the contact layer. Their gel forming characteristics and antimicrobial properties can help maintain a fresh and dry interface between the body and the absorbent materials. In addition, they can help reduce leakage and prevent contamination and the transmission of infectious diseases.

\section{Tissue engineering}

Both alginate and chitosan fibers have excellent biocompatibility and cell affinity, making them ideal materials for the preparation of scaffolds in tissue engineering. Shao \& Hunter ${ }^{21}$ developed a process for combining the properties of alginate and chitosan by placing the swollen calcium alginate fiber from the wet spinning process into an aqueous chitosan solution. Once the chitosan is absorbed onto the surface of the calcium alginate fibers, the resultant poly blend fibers were then freeze dried to prepare fibrous materials that can be used as scaffold material in tissue engineering. Figure 6 shows an illustration of the applications of alginate and chitosan fibers in tissue engineering.

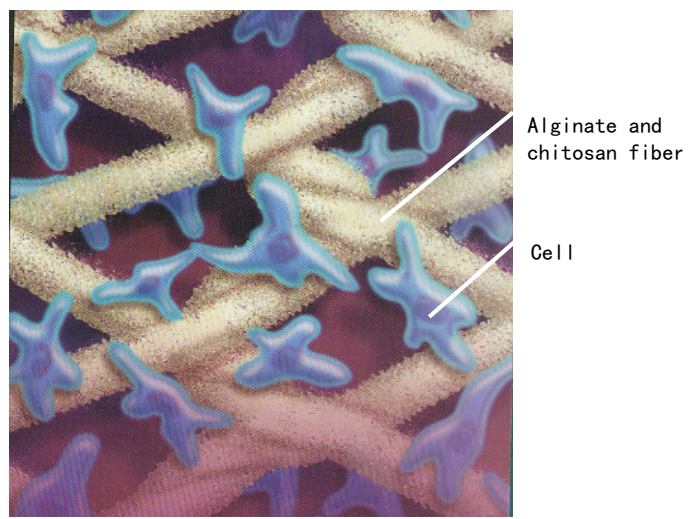

Figure $\mathbf{6}$ An illustration of the applications of alginate and chitosan fibers in tissue engineering.

\section{Summary}

Both alginate and chitosan have many novel properties, making them useful as excellent biomaterials. When made into fibers, alginate and chitosan can be further processed into woven, knitted, nonwoven and other two or three dimensional structures. Alginate and chitosan have on their molecules acid and amine groups respectively, making it easy for them to form novel materials with metal ions, or through chemical modifications to achieve better performances. Alginate and chitosan fibers have already been successfully used as raw materials for making advanced wound dressings, hygiene and cosmetic products. It can be foreseen that more and more novel biomedical products can be developed from alginate, chitosan and their composite materials.

\section{Acknowledgements}

None.

\section{Conflict of interest}

Author declares there is no conflict of interest in publishing the article.

\section{References}

1. Qin Y. Medical Textile Materials. 1st ed. New York: Elsevier; 2016. p. 264.

2. Agboh OC, Qin Y. Chitin and chitosan fibers. Polymers for Advanced Technologies. 1997;8(6):355-365.

3. Qin Y. Alginate fibers: an overview of the production processes and applications in wound management. Polymer International. 2008;57(2):171-180

4. Qin Y. The gel swelling properties of alginate fibers and their application in wound management. Polymers for Advanced Technologies. 2008;19(1):6-14.

5. Bixler HJ, Porse H. A decade of change in the seaweed hydrocolloids industry. J Appl Phycol. 2011;23(3):321-335. 
6. FAO. Fishery and Aquaculture Statistics. 2016.

7. Speakman JB, Chamberlain NH. The production of rayon from alginic acid. J Society of Dyers \& Colourists. 1944;60(10):264-272.

8. Dong J. Clinical observation of the promotion of wound healing by chitosan dressing. Chinese Journal of Nosocomiology. 2011;21(5):918-919.

9. Tamura H, Tsuruta Y, Tokura S. Preparation of chitosan-coated alginate filament. Materials Science \& Eng C. 2002;20(1-2):143-147.

10. Knill CJ, Kennedy JF, Mistry J, et al. Alginate fibers modified with unhydrolysed and hydrolysed chitosans for wound dressings. Carbohydrate Polymers. 2004;55(1):65-76.

11. Miraftab M, Kennedy JF, Groocock R. Wound management fibers. USP2008/0097001 A1. 2008.

12. Majima T, Funakosi T, Iwasaki N, et al. Alginate and chitosan poly ion complex hybrid fibers for scaffolds in ligament and tendon tissue engineering. J Orthop Sci. 2005;10(3):302-307.

13. Qin Y. Gel swelling properties of alginate fibers. J Applied Polymer Sci. 2004;91(3):1641-1645.

14. Qin Y. The characterization of alginate wound dressings with different fiber and textile structures. J Applied Polymer Science. 2006;100(3):2516-2520.
15. Haug A, Smidsrod O. The Effect of Divalent Metals on the Properties of Alginate Solutions. II. Comparison of Different Metal Ions. Acta Chemica Scandinavica. 1965;19(2):341-351.

16. Qin Y. The ion exchange properties of alginate fibers. Textile Research J. 2005;75(2):165-168.

17. Wijesinghe WAJP, Jeon YJ. Biological activities and potential cosmeceutical applications of bioactive components from brown seaweeds: a review. Phyto chem Rev. 2011;10(3):431-443.

18. Thomas S. Alginate dressings in surgery and wound management--Part 1. J Wound Care. 2000;9(2):56-60.

19. Thomas S. Alginate dressings in surgery and wound management--Part 2. J Wound Care. 2000;9(3):115-119.

20. Thomas S. Alginate dressings in surgery and wound management--Part 3. J Wound Care. 2000;9(4):163-166.

21. Shao X, Hunter CJ. Developing an alginate/chitosan hybrid fiber scaffold for annulus fibrosus cells. J Biomed Mater Res A. 2007;82(3):701-710. 